\title{
Les techniques de construction navale aux Maldives originaires d'Asie du Sud-Est
}

About boat-building techniques of the Maldives of Southeast Asian origin

De las técnicas de la construcción naval en las Maldivas, oriundas del Asia del sudeste

Pierre-Yves Manguin

\section{CpenEdition}

\section{Journals}

Édition électronique

URL : https://journals.openedition.org/tc/276

DOI : $10.4000 /$ tc. 276

ISSN : $1952-420 X$

Éditeur

Éditions de l'EHESS

\section{Édition imprimée}

Date de publication : 1 janvier 2001

Pagination : 21-47

ISSN : 0248-6016

\section{Référence électronique}

Pierre-Yves Manguin, «Les techniques de construction navale aux Maldives originaires d'Asie du SudEst », Techniques \& Culture [En ligne], 35-36 | 2001, mis en ligne le 07 septembre 2012, consulté le 29 septembre 2022. URL : http://journals.openedition.org/tc/276 ; DOI : https://doi.org/10.4000/tc.276

Ce document a été généré automatiquement le 29 septembre 2022.

Tous droits réservés 


\title{
Les techniques de construction navale aux Maldives originaires d'Asie du Sud-Est
}

\author{
About boat-building techniques of the Maldives of Southeast Asian origin \\ De las técnicas de la construcción naval en las Maldivas, oriundas del Asia del \\ sudeste
}

Pierre-Yves Manguin

1 Une mission effectuée en mars 1988 dans l'archipel des Maldives m'a permis de mener une étude rapide, mais relativement exhaustive, des divers chantiers de construction navale traditionnelle de l'atoll de Maafushi, immédiatement au sud de l'île capitale de Male, et des «atolls du Nord» Hinnavaru, Alifushi et Iguraidhoo². On rappellera ici brièvement que la République des Maldives est constituée d'un millier d'atolls coralliens, dont deux cents environ sont habités, situés au cœur de l'océan Indien, entre Io Sud et $8^{\circ}$ Nord de latitude. Ses 200000 habitants parlent une langue du groupe indoeuropéen, le dhivehi, apparentée à celle de Sri Lanka. Ils pratiquent tous aujourd'hui la religion musulmane; il y a environ un millénaire, l'archipel a cependant connu une phase d'indianisation, moins approfondie que celle de l'Asie du Sud-Est et qui a laissé dans les atolls méridionaux nombre de vestiges de monuments bouddhiques ${ }^{3}$. Un quart d'entre eux habitent Male, la capitale, qui occupe la totalité de la surface d'un des atolls $\mathrm{du}$ centre de l'archipel. La noix de coco et le poisson, obtenus sur place, constituent l'essentiel de leur régime alimentaire, avec le riz importé ${ }^{4}$. À la fin des années quatrevingt, une vaste flotte de bateaux traditionnels, les dhoni (photos 1 et 2), marchant à la voile et au moteur, assurait encore une part importante des activités économiques des Maldives : pêche à la bonite et communications entre les atolls ${ }^{5}$.

2 Les plus fortes concentrations de chantiers construisant «à l'ancienne " ont été trouvées sur les atolls d'Alifushi et de Iguraidhoo. À l'exception du State Shipyard d'Alifushi, qui utilisait déjà des techniques de construction occidentales, l'ensemble de ces embarcations était encore fabriqué selon des procédés et avec des matériaux 
traditionnels (les charpentiers du State Shipyard construisaient tous des dhoni «à l'ancienne » en dehors de leurs heures de service). Pendant ce séjour, tant à Maafushi que dans les Atolls du Nord, je n'ai observé aucune différence marquante dans les techniques utilisées pour la construction des dhoni. Ceci confirme les affirmations des constructeurs interrogés dans les atolls et des informateurs compétents rencontrés à Male : tous les dhoni des Maldives sont construits en suivant une technique homogène, unique. Je ne peux confirmer cette observation en ce qui concerne les Atolls du Sud, lesquels n'ont pu être visités. Cependant, on m'a affirmé à plusieurs reprises que les dhoni y ont une structure homologue et y sont assemblés selon des procédés identiques; la seule différence notoire n'est pas structurelle: les Atolls du Sud ayant des lagons plus profonds que ceux du Nord, ils permettent l'usage de dhoni disposant d'un plus grand tirant d'eau (photo 2). Les informations présentées dans ce bref article ont donc toutes les chances d'être valables pour l'ensemble de l'Archipel des Maldives.

\section{La structure et la construction d'un dhoni}

Seul le bois de cocotier (Cocos nucifera) est disponible aux Maldives en quantités substantielles. Il entre de ce fait pour une part essentielle dans la construction des dhoni, en dépit de la mauvaise réputation qu'il a auprès des charpentiers de marine ${ }^{6}$. Sur les chantiers que nous avons visités, des bois différents étaient utilisés pour les seules chevilles, faites dans du kuredhi (le buisson Pemphis acidula) et pour certaines parties structurelles de la coque, dont surtout la quille, pour laquelle on utilisait le bois plus dur de Bruguiera cylindrica, l'un des rares grands arbres de la mangrove locale (kadoo en dhivehi) ${ }^{7}$. Tous les cordages employés sur les bateaux sont faits de fibres de cocotier, le fameux « coir » des Maldives, qui est, aujourd'hui comme par le passé, l'une des productions importantes de l'archipel ${ }^{8}$.

Le tronc de cocotier est d'abord immergé dans l'eau de mer pendant une durée pouvant atteindre trois semaines, pour que les planches qui en sont extraites puissent être cintrées à la vapeur, si le besoin s'en fait sentir, en les chauffant au-dessus d'un feu. Les troncs sont habituellement débités à la hache ou sciés en long pour donner deux planches épaisses (de $10 \mathrm{~cm}$ environ), longues de $2 \mathrm{~m}$ et larges de $20 \mathrm{~cm}$ à peu près. Ces planches sont ensuite débitées avec herminettes et haches de dimensions diverses pour leur donner leur forme définitive -couples, baux ou bordés-: un bordé destiné à un dhoni de $15 \mathrm{~m}$ de long aura ainsi une épaisseur finale de $6 \mathrm{~cm}$ environ (photo 3 ). 


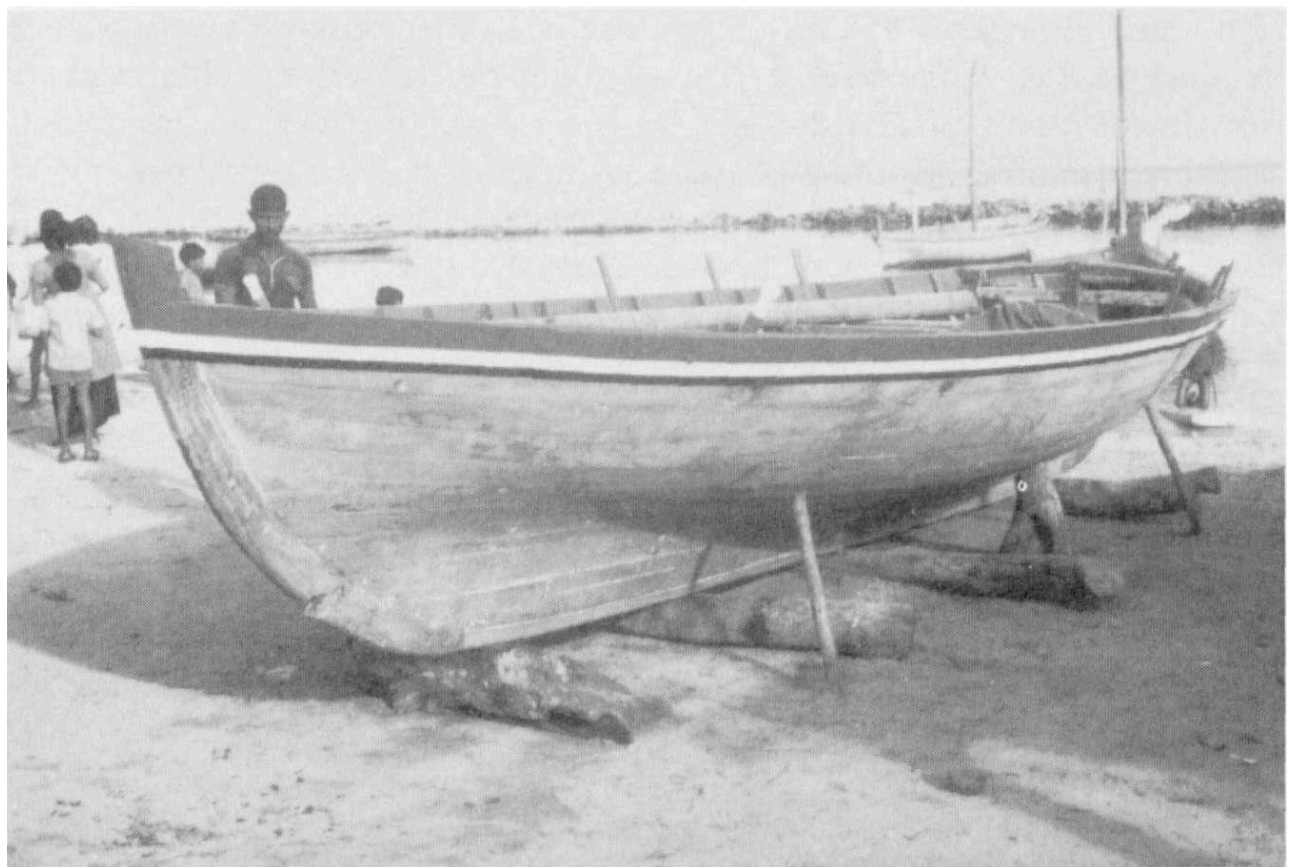

Photo 1. Petit dhoni au sec à Hinnavaru

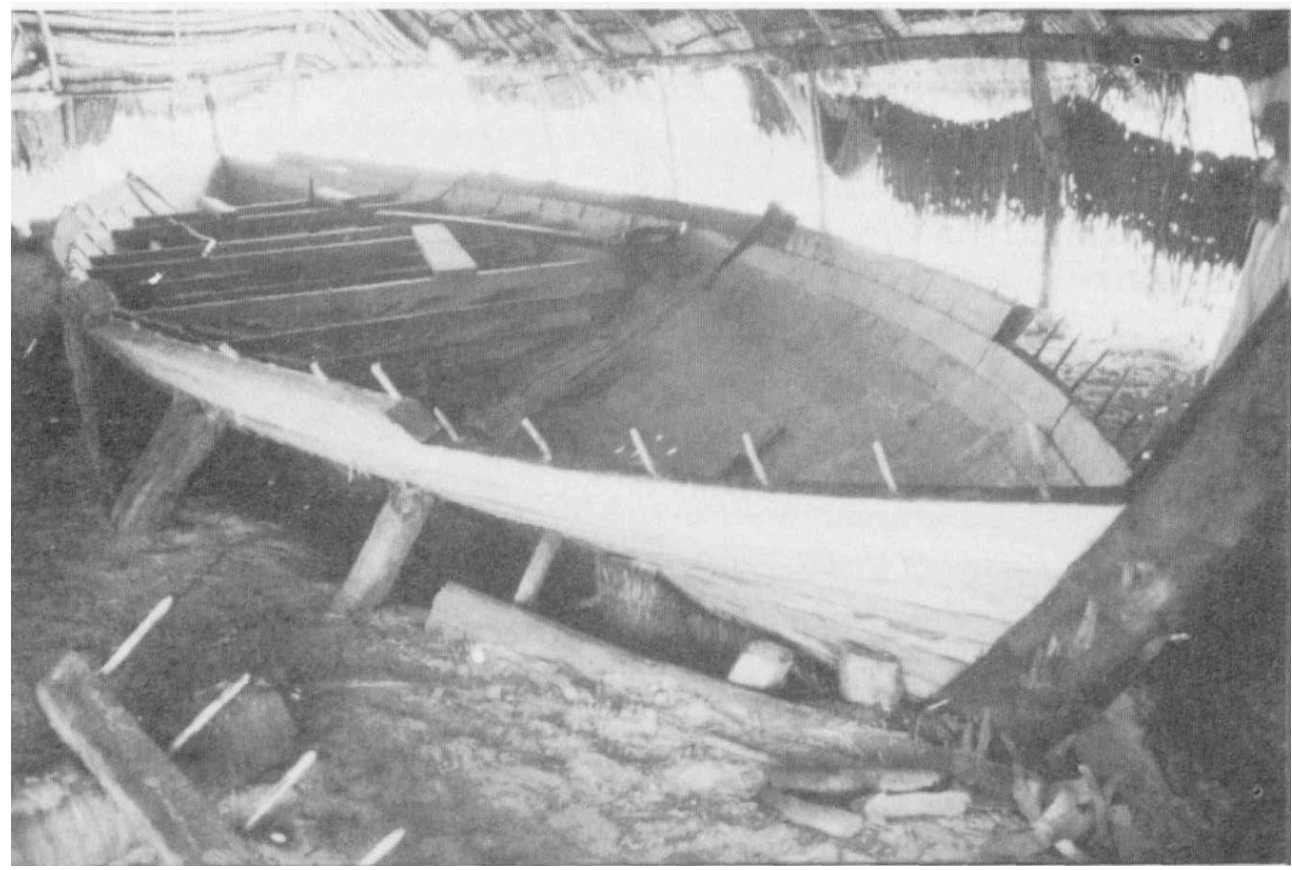

Photo 2. Grand dhoni en construction à Alifushi (construction sans blocs) 


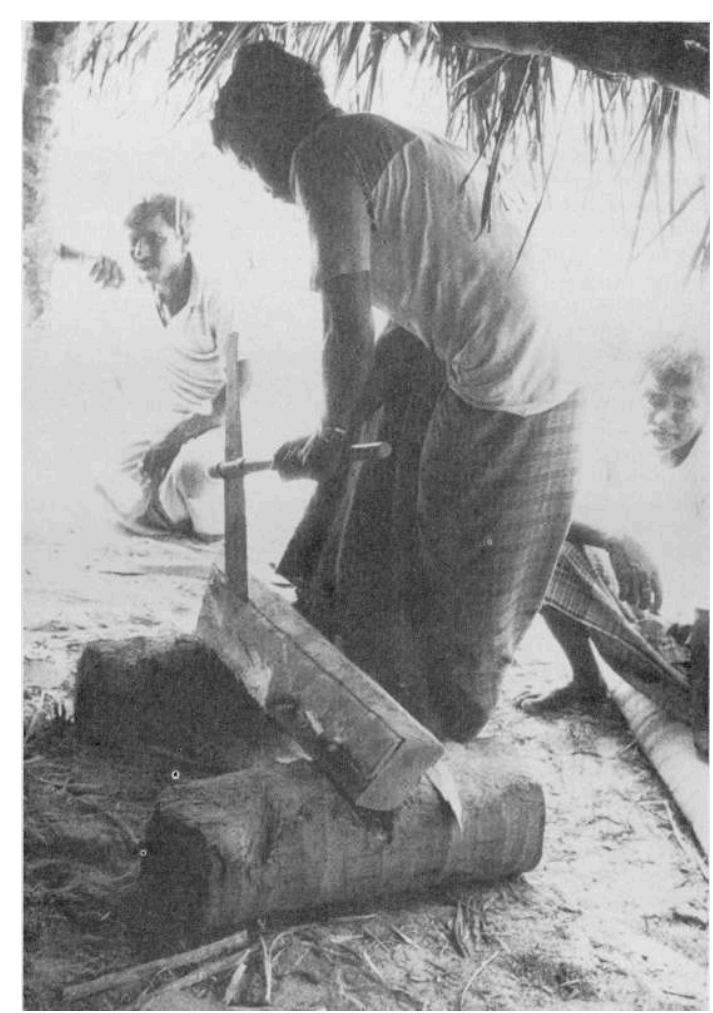

Photo 3. Débitage d'un bordé à la hache

5 Tous les dhoni sont construits, fondamentalement, selon le principe dit de «shell first » : la coque est assemblée avant que les couples n'y soient insérés; mais, comme c'est le plus souvent le cas, le principe est aménagé : les varangues et quelques demicouples sont de fait mis en place avant les virures les plus hautes (photo 4).

6 La première virure est assemblée à feuillure à la quille et aux pièces d'étrave et de poupe, préalablement jointes entre elles par des chevilles. Des chevilles en bois sont de même utilisées pour assembler le galbord à la quille et aux pièces d'étrave et de poupe, puis les bordés successifs entre eux. L'étanchéité entre les virures est obtenue par deux opérations successives (dont on verra le détail plus loin) : en premier lieu, les surfaces en contact des deux bordés (les cans) sont rendues aussi jointives que possible ; puis de fines lamelles d'écorce de noix de coco sont insérées entre les virures, autour des chevilles, avant que l'assemblage ne soit bloqué en place.

7 Les couples sont insérés dans la coque préalablement assemblée, à des intervalles variables (de $25 \mathrm{~cm}$ à $80 \mathrm{~cm}$ d'écart, selon la longueur du dhoni ou selon le nombre de baux qui seront implantés ${ }^{9}$. Les couples principaux, de plus forte section, sont disposés à intervalles réguliers: c'est à leur hauteur que les baux seront mis en place. Les varangues sont assemblées aux allonges par des joints à sifflet. Entre ces couples principaux, des demi-couples plus légers sont insérés. À la fin des années quatre-vingt, les bordés des dhoni que j'ai vu construire étaient toujours liés aux couples par des rivets en cuivre. Cependant, aux dires des constructeurs locaux, des chevilles en bois étaient encore utilisées peu de temps auparavant; j'ai pu vérifier cette affirmation: quelques vieux dhoni abandonnés sur les plages avaient leurs bordés ainsi liés par des chevilles tronconiques, bloquées en place par l'insertion dans leur tête d'un petit coin en bois, depuis l'extérieur de la coque (photo 5). 
8 On pourrait retrouver les caractéristiques décrites jusqu'ici sur la plupart des embarcations traditionnelles de l'Asie du Sud-Est ou de l'océan Indien. Il est cependant d'autres traits structurels des dhoni qui vont nous permettre de les distinguer des traditions de la construction navale du sous-continent indien ou du Moyen-Orient et d'établir des rapports étroits avec celles de l'Asie du Sud-Est.

En premier lieu, des rangées de baux (ihi) sont insérées dans la coque à intervalles réguliers (tous les deux ou trois couples), selon le type de dhoni, sa taille ou l'usage auquel il est destiné. Chaque rangée compte de trois à cinq baux superposés. Le bau inférieur de chacune d'elles est disposé à la hauteur de la cinquième ou de la sixième virure à compter de la quille. Dans les modèles de dhoni les plus anciens (ceux qui, hors d'usage, ont été abandonnés sur les plages), des blocs de bois rectangulaires, épais d'environ $4 \mathrm{~cm}$, ont été réservés à la surface interne des bordés lorsque ceux-ci ont été débités, à l'endroit précis où chacun des baux transversaux est assemblé tant aux bordés qu'aux couples (photos 5 à 7). Ces blocs en bois continuaient en 1988 d'être réservés sur la face interne des bordés de la plupart des dhoni que j'ai vu construire ; ils y apparaissaient cependant sous forme de vestiges, au point d'être parfois tout juste apparents (moins de $1 \mathrm{~cm}$ d'épaisseur), mais sont toujours désignés par les termes fothi ou gandu. De fait, les constructeurs m'ont affirmé qu'ils les conservaient dans ces cas par seul goût de la tradition, alors même qu'ils ne jouaient plus aucun rôle (on verra plus loin l'explication de cette anomalie) (photo 8). Sur les dhoni destinés à la pêche à la bonite, ces rangées transversales de baux sont transformées en cloisons étanches par un doublage en planches, de façon à y créer un compartiment étanche dans lequel sont conservés les appâts vivants.

10 Il faut signaler enfin que des pièces de bois verticales, joliment tournées sur les bateaux les plus anciens, sont insérées à intervalles variables entre les baux horizontaux. Ces pièces verticales portent en dhivehi le nom de haki, dont le sens premier décrit les crampons qui permettent à la liane du bétel de s'accrocher au tronc qu'elle enserre; cette fonction n'est pas sans rappeler celle de ces pièces en bois qui rendent les baux solidaires les uns des autres (on verra plus loin en quoi cette fonction est essentielle dans la structure d'ensemble des plus anciens dhoni) (photo 5).

11 Il nous faut aussi décrire ici en détail le processus suivi pendant la construction d'un dhoni, et en particulier lors de l'assemblage de deux bordés successifs, car il fait appel à des procédures en tous points comparables à celles que l'on peut observer de nos jours en Asie du Sud-Est insulaire, et dont la description a été relevée à une époque historique.

Dès lors qu'un bordé a été mis en place définitivement et assemblé avec celui qui le précède, des orifices sont forés dans son épaisseur, à partir du can supérieur, pour l'insertion de chevilles (photo 9). L'outil à forer parfois encore utilisé à la fin des années quatre-vingt était muni de mèches à cuiller et entraîné à la corde (il faut donc trois hommes pour l'actionner). Des chevilles provisoires, de diamètre légèrement inférieur à celui de ces orifices, y sont alors insérées. Le bordé suivant a entre-temps été mis à la forme exacte qui lui revient sur la coque, au moyen de haches et d'herminettes; l'ajustage s'est fait par approximations successives ; des orifices ont enfin été forés dans son épaisseur où les chevilles provisoires vont être engagées. Lorsque la forme du nouveau bordé est bien ajustée, il est mis en place et maintenu provisoirement en compression. 


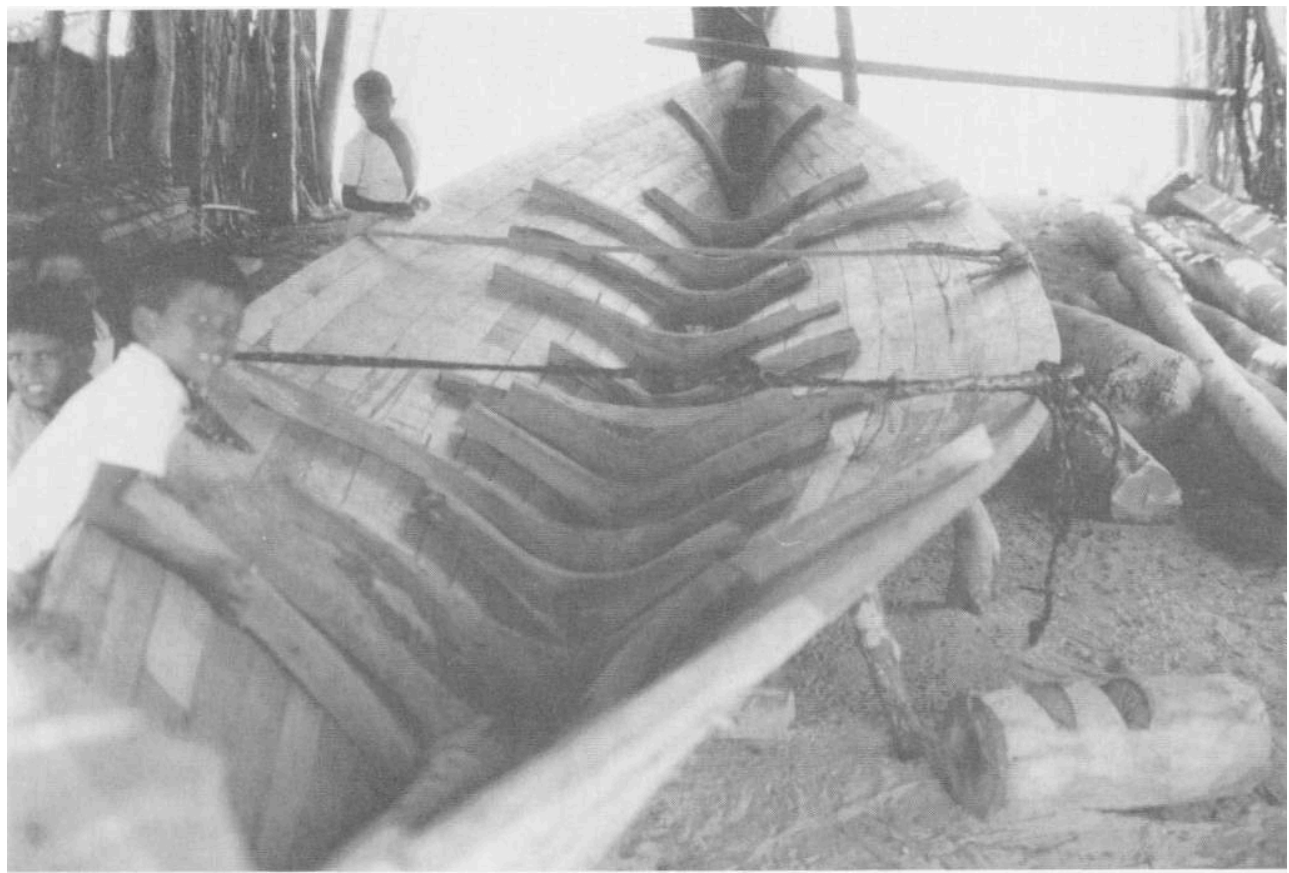

Photo 4. Phase intermédiaire de la construction de la coque : après 1 assemblage des virures du fond, les varangues ont été mises en place on remarque les cordages qui tiennent le tout en compression

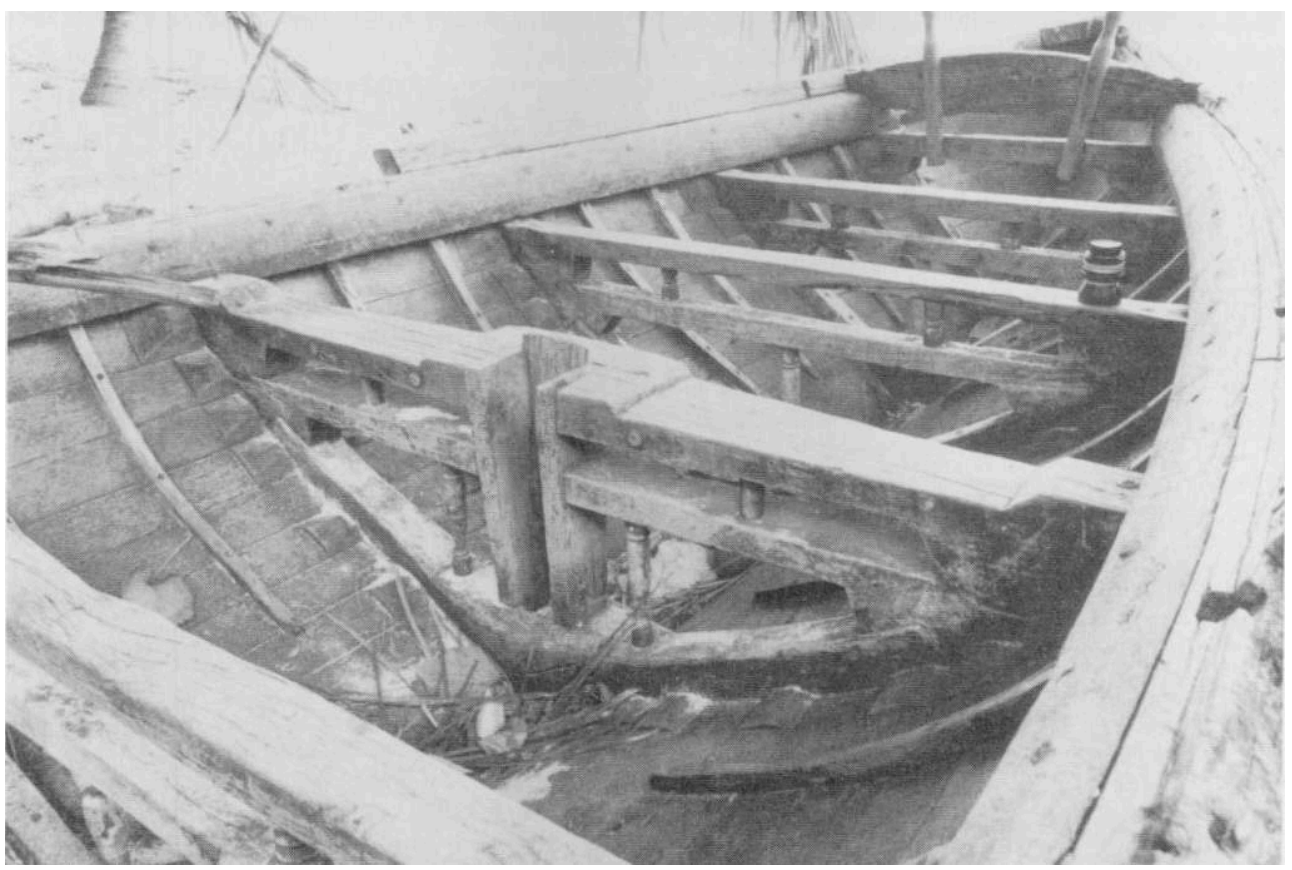

Photo 5. Ancien dhoni de petite taille à l'abandon : on distingue les rangées de blocs (fothi) réservés sur la face interne des bordés, les solides couples et baux qui leur sont associés (l'ensemble du premier plan est particulièrement épais, car il servait d'emplanture au mât), les petits demi-couples insérés entre les baux; on distingue aussi les pièces de bois tourné (hakı) qui relient les baux d'une même rangée entre eux 


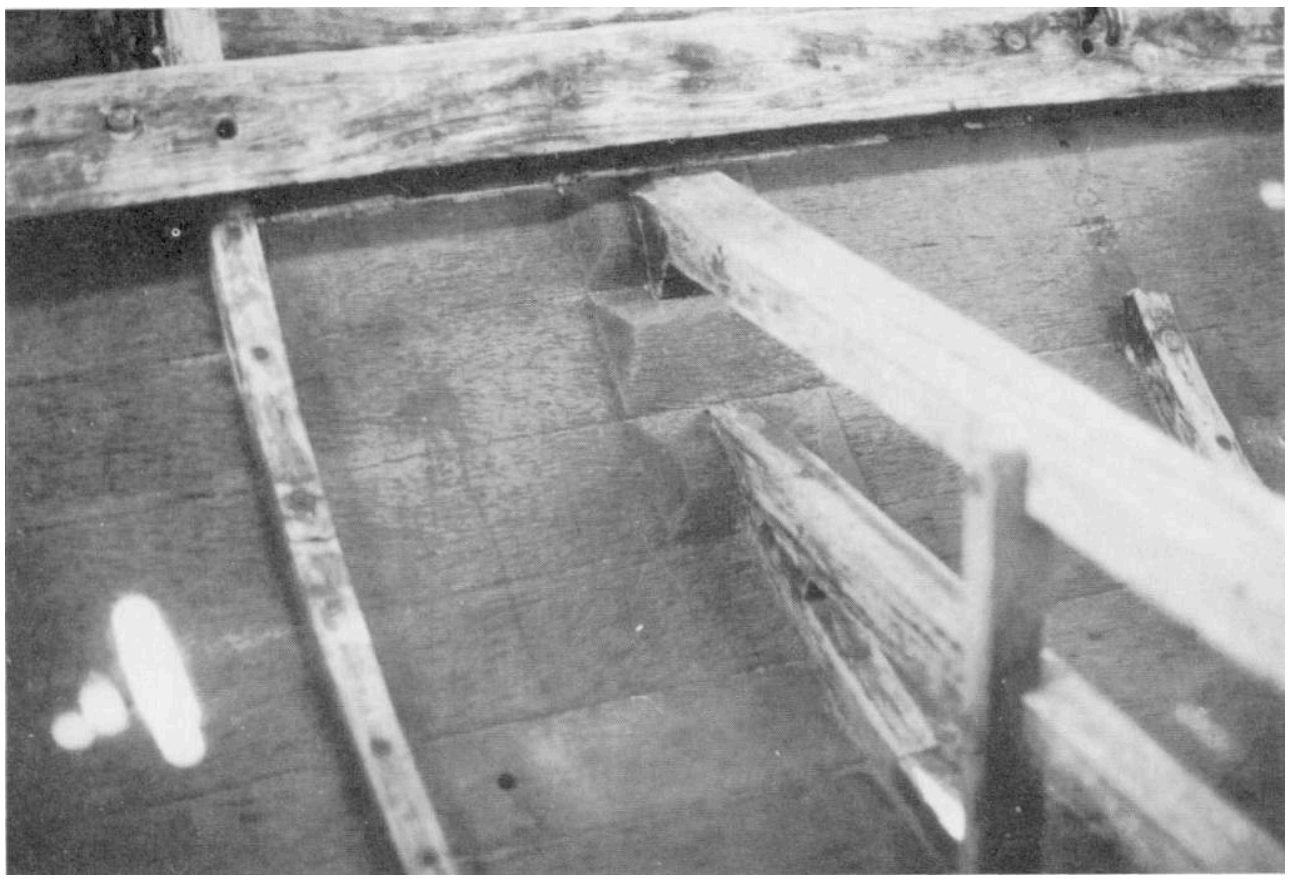

Photo 6. Blocs (fothi) servant d'appui aux baux sur un petit dhoni ; on remarque la forte section des baux et la minceur des demi-couples

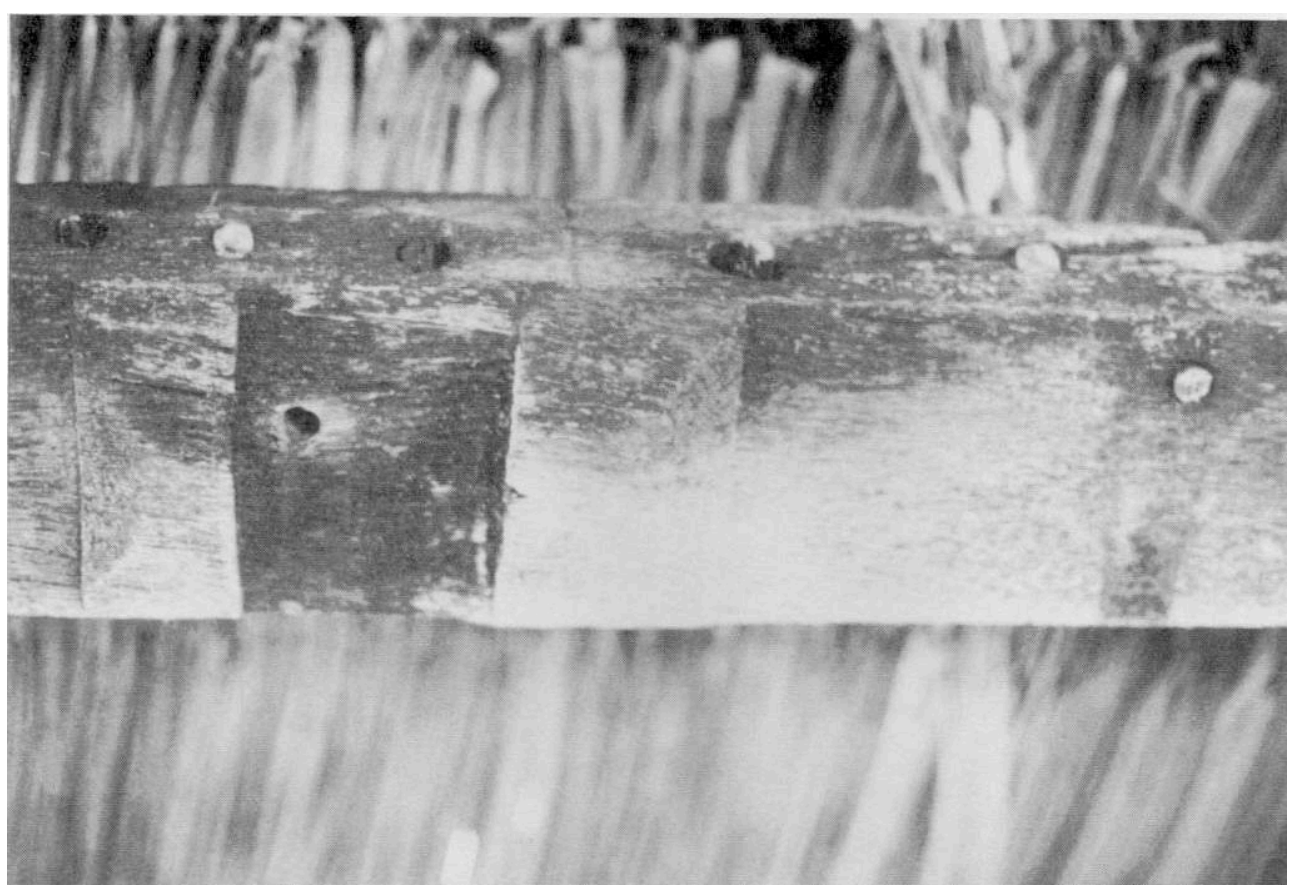

Photo 7. Bordé avec bloc en bois d'un ancien dhoni (démonté) : on distingue, à gauche, l'emplacement réservé pour le couple dans le bloc avec le trou de la cheville qui le joignait à ce bordé ; à droite, la trace laissée par un demi-couple et les chevilles, très rapprochées, sur la surface supérieure du bordé 


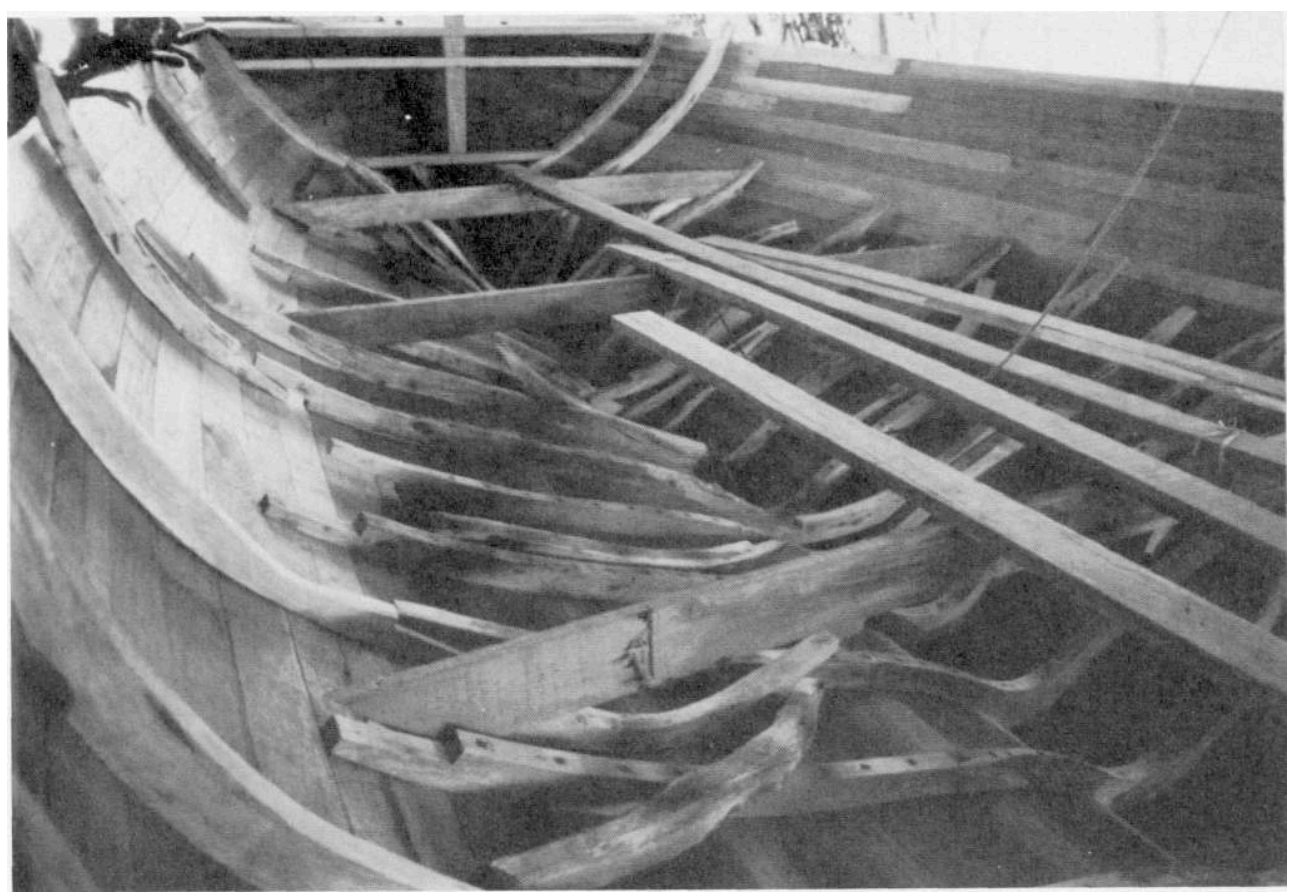

Photo 8. Coque presque terminée d'un dhoni sans blocs : les couples sont nombreux et de forte section; les baux, rares, ne sont pas encore tous en place

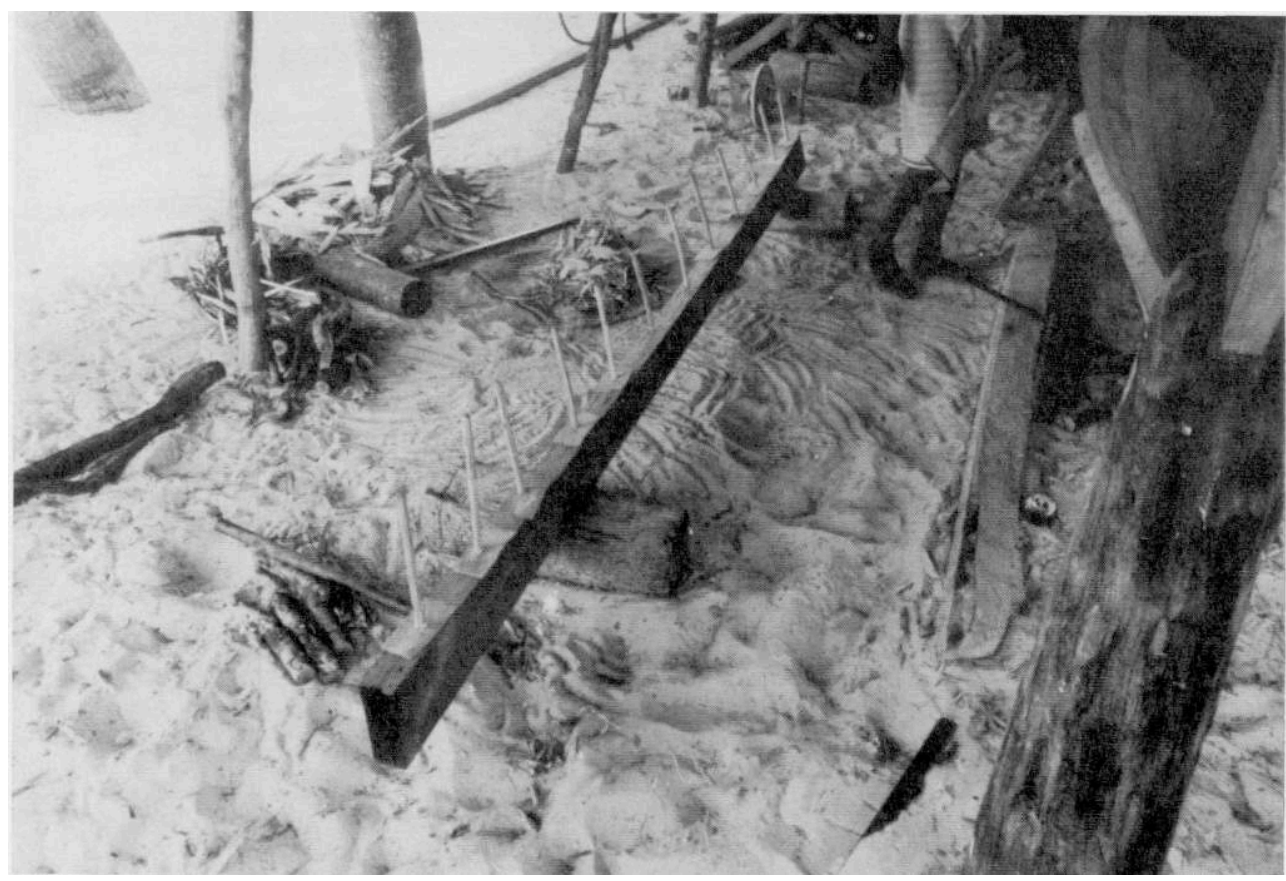

Photo 9. Préparation d'un bordé avec ses chevilles définitives : on distingue les morceaux d'écorce de noix de coco engagés autour de chacune d'elles

Un petit outil métallique bifide (kalihi), équivalent du trusquin des charpentiers français, permet alors, en suivant précisément l'arête du bordé inférieur, déjà définitivement en place, de tracer une ligne parallèle sur la face externe du nouveau bordé, maintenu provisoirement fixé. Ce dernier est alors enlevé à nouveau et, en suivant cette ligne, un charpentier peut, avec une herminette, ajuster précisément sa surface inférieure de façon à obtenir un contact satisfaisant entre les deux bordés. Un bon charpentier de marine peut se passer du trusquin et donc faire l'économie de cette 
phase du processus, en se fiant entièrement à son œil pour ajuster les deux virures ; j'en ai été le témoin à Maafushi.

Le nouveau bordé est alors à nouveau démonté et de la suie de noix de coco est passée sur la face supérieure du bordé en place. Le nouveau bordé est encore une fois remonté et, à coups de masse, inséré en place. Les parties saillantes de la face inférieure du nouveau bordé vont être noircies par la suie, laissant en blanc les surfaces où le contact n'est pas encore parfait. Le charpentier, après avoir enlevé derechef le nouveau bordé, va araser à l'herminette les parties noircies par la suie. Une succession d'essais va ainsi progressivement assurer un contact parfait entre les deux bordés. Lorsque le charpentier estime que celui-ci est enfin obtenu, il remplace les chevilles provisoires par des chevilles définitives, d'un diamètre supérieur, qui devront être martelées avec force pour être insérées. De fines lamelles d'écorce de noix de coco sont placées sur la surface du bordé inférieur et maintenues en place par les chevilles qui les traversent: elles gonfleront lorsqu'elles seront humides, achevant d'assurer l'étanchéité de la coque. À ce stade, le nouveau bordé peut être inséré définitivement, à coups de masse. Il ne pourra plus être démonté. L'assemblage est parfois bloqué en place par de petites contre-chevilles insérées depuis l'intérieur de la coque (photo 10).

Aucun calfatage ne sera nécessaire avant la mise à l'eau; les cans des deux bordés consécutifs n'ont d'ailleurs pas été aménagés pour permettre cette opération. Par contre, une vieille coque dont les bordés commencent à se disjoindre pourra plus tard être calfatée avec un mélange d'huile de requin et de noix de Calophyllum inophyllum (funa en dhivehi).

Il est important de noter enfin que, pendant l'assemblage des bordés suivant le processus décrit ci-dessus, de forts cordages enserrent en plusieurs points la coque : ils sont fortement tendus avec des tourniquets pour assurer la position des virures déjà en place, mais surtout pour maintenir la structure de la coque en compression jusqu'à ce que toutes les virures, les couples et les baux aient trouvé leur place et soient rendus solidaires les uns des autres par des chevilles ou des rivets. La coque achevée se maintient alors d'elle-même en compression et les cordages qui l'insèrent peuvent être enlevés. 


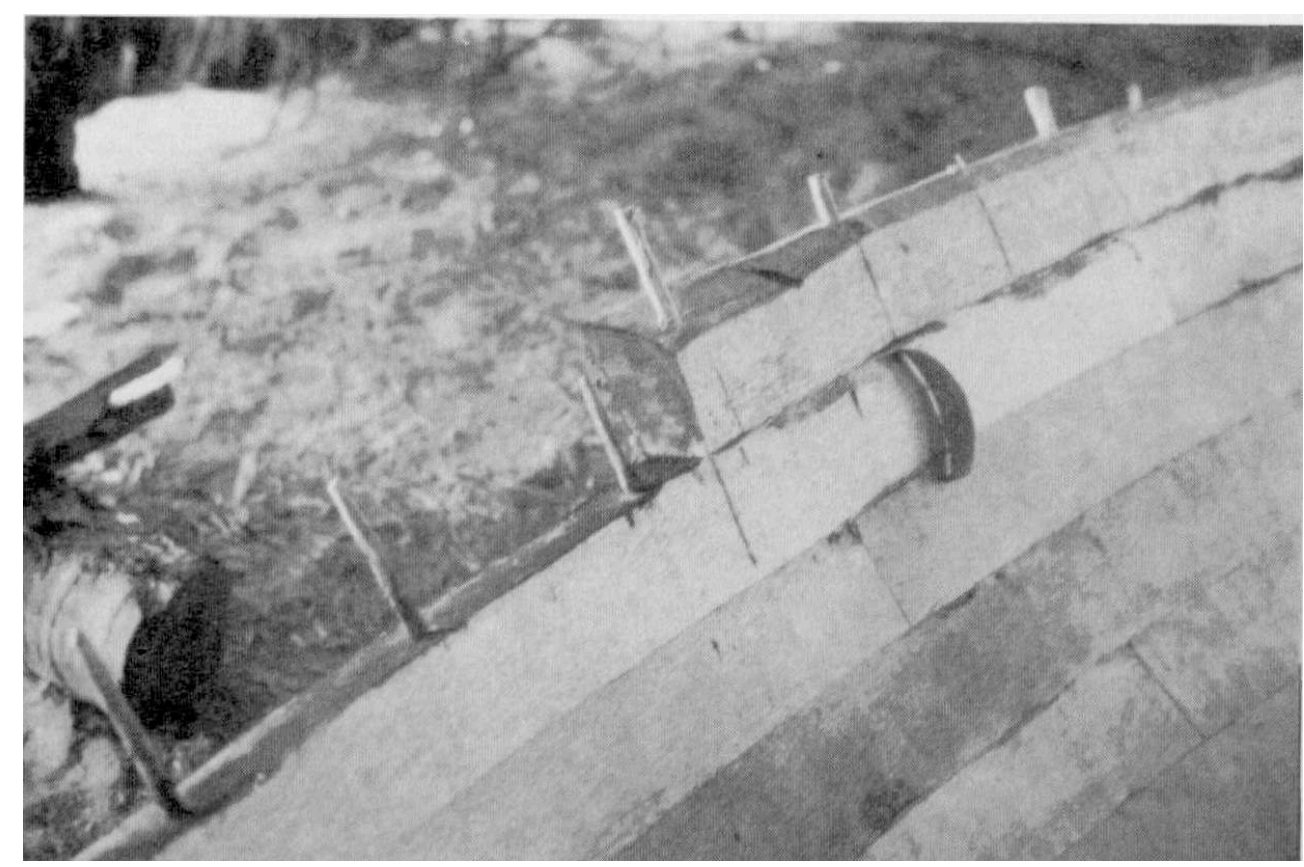

Photo 10. Assemblage progressif des bordés d'un dhoni sans blocs : le bordé le plus haut est en place. On distingue les fragments d'écorce de noix de coco qui ont été insérés entre les deux bordés successifs; le can du bordé inférieur est passé au noir de suie et les chevilles provisoires sont insérées pour les essais de mise en place du bordé suivant

\section{L'évolution d'une tradition technique}

Pendant ce séjour trop bref aux Maldives, j'ai observé un nombre suffisant de dhoni, tant à l'état achevé qu'à divers stades de leur construction, pour pouvoir détecter certaines variantes pertinentes des techniques décrites ci-dessus; toutes cependant demeuraient clairement dans les limites d'une unique tradition de construction navale.

Je voudrais donc proposer ici -de façon très simplifiée- un schéma évolutif de la construction des dhoni, afin de tenter tout à la fois d'expliquer l'existence même de ces variantes et de fournir des éléments de comparaison avec des techniques analogues observées en Asie du Sud-Est insulaire.

Stade I. Lorsque de forts blocs rectangulaires sont réservés sur la face interne des bordés, ils jouent encore un rôle vital comme point de ligature entre baux, couples et bordage. Sur de tels bateaux, les couples sont relativement écartés $(80 \mathrm{~cm}$ d'écart sur un dhoni de $10 \mathrm{~m}$ ) et de section faible, mais les rangées de baux transversaux sont nombreuses et les baux eux-mêmes, de forte section : ils compensent donc la relative faiblesse des couples et ce sont eux qui contribuent plus largement au renforcement de la structure de la coque.

Stade II. Lorsque ces blocs n'apparaissent plus que sous forme de vestiges, les couples sont plus nombreux ( $25 \mathrm{~cm}$ d'écart sur un dhoni de $15 \mathrm{~m}$ ). Les rangées de baux sont en moins grand nombre et leur rôle structurel diminue au profit des couples.

Stade III. Sur certains dhoni, les blocs de bois ont totalement disparu; les couples sont alors très rapprochés et la fréquence des rangées de baux diminue encore. Ils avaient totalement disparu sur un seul des dhoni observés, mais les couples y étaient de section nettement plus forte que d'habitude; en fait, ils restaient seuls pour renforcer la coque. 
incursion dans l'histoire de la construction navale aux Maldives va nous permettre de mieux appréhender ce schéma évolutif, en mettant en évidence un état antérieur au Stade I, que l'on ne peut plus observer de nos jours. Il est à noter cependant que l'évolution ainsi détectée n'a pas nécessairement eu lieu de façon strictement linéaire, ou chronologique, puisque les trois stades décrits ci-dessus semblent bien coexister aux Maldives, parfois au sein du même chantier naval.

\section{Données historiques}

Trois témoignages précis, recueillis aux $\mathrm{XV}^{\mathrm{e}}$ et $\mathrm{XVI}^{\mathrm{e}}$ siècles, nous fournissent des données techniques d'ensemble sur la construction navale aux Maldives, dont la cohérence est tout à fait remarquable.

Le Chinois Ma Huan, qui accompagnait dans ses expéditions maritimes le fameux Amiral Zheng He, a visité les Maldives en 1413 et en 1421; il nous a laissé une description précise des navires qu'il y a vu construire : "Jamais ils n'utilisent de clous [en fer]; ils ne font que forer des trous et utilisent toujours leurs cordes [de coir] pour lier [les bordés] ensemble, en combinaison avec des chevilles en bois ».

Un siècle plus tard, le chroniqueur portugais Gaspar Correia -toujours le plus précis de ses contemporains lorsqu'il s'agit de faits techniques- décrit les embarcations des Maldives observées en 1503 en termes semblables: "Ils sont fabriqués en bois de cocotier et assemblés avec des chevilles en bois, sans qu'aucun clou ne soit utilisé ».

Légèrement plus tard, au XVI ${ }^{\mathrm{e}}$ siècle, Duarte Barbosa, bon connaisseur de la région, confirme que les habitants des Maldives «[...] construisent de nombreux et grands navires en bois de palmier, liés avec des fibres, car ils n'ont aucun autre bois de construction $»^{10}$.

Si l'on fait exception d'un point capital, sur lequel on reviendra, ces trois descriptions confirment les données fournies par l'observation moderne. Ainsi, l'usage des chevilles, attesté en 1988 sur les seuls dhoni les plus anciens, est noté par à la fois par Ma Huan et par Correia. Les deux auteurs du XVI ${ }^{e}$ siècle mentionnent l'usage dominant du bois de cocotier.

Toutefois, comment doit-on interpréter l'information, donnée par Ma Huan et par Barbosa, selon laquelle les bordés des bateaux des Maldives sont assemblés, outre les chevilles, avec des liens en fibre végétale? Aucun des témoignages modernes sur les Maldives ne fait état de navires dont les éléments structurels auraient été cousus ou liés entre eux (alors même que les bordés cousus sont caractéristiques des navires du Moyen-Orient comme de l'Inde). Aux Maldives même, aucun des informateurs interrogés, charpentiers de marine ou érudits locaux férus de témoignages plus anciens, ne se souvenait d'avoir jamais entendu mentionner un tel mode d'assemblage (alors que le chevillage en bois des bordés aux couples, aujourd'hui toujours remplacé par des rivets en cuivre, est encore bien présent dans les mémoires, comme on l'a vu plus haut).

Il existe pourtant une donnée linguistique qui semble indiquer un usage ancien de liens pour assembler les bateaux locaux. En effet, le mot dhivehi employé pour décrire l'action de construire un bateau est banun, qui signifie littéralement «attacher » ou "lier», avec une corde -l'action de construire une maison, par exemple, est décrite par un autre terme, hedun, qui, s'il est utilisé pour les bateaux, ne peut signifier que 
« réparer ». L'usage prolongé de ce terme aux implications techniques précises dans le monde des charpentiers de marine, est donc la deuxième indication que l'on ait d'un emploi ancien des ligatures en fibres végétales dans la construction navale des Maldives. Pour avoir une confirmation supplémentaire de l'existence passée d'une telle technique d'assemblage, il nous faut d'abord faire le détour par l'Asie du Sud-Est ancienne et moderne et démontrer les origines sud-est asiatiques des traditions des Maldives.

\section{Analogies avec les techniques de l'Asie du Sud-Est} jours, naviguent parfois sur l'océan Indien et les mers de l'Asie du Sud-Est, est d'avoir des bordés assemblés entre eux au moyen de cordages faits de fibres végétales. Cependant, il faut distinguer au sein de cette grande famille deux traditions distinctes de mode de ligature : coques cousues et coques liées.

31 Les coques cousues caractérisent les navires construits au Moyen-Orient ou en Inde: par liaison « cousue », nous entendons un assemblage réalisé, avec une fil et des points de couture continus, de trou en trou, le long de chaque virure, sans interruption de la proue à la poupe (comme on le ferait pour deux pièces de tissu) ; ces "coutures " traversent le bordé de part en part et sont donc visibles de l'extérieur. Les bordés peuvent être en outre maintenus en place par des chevilles.

32 Je préfère utiliser le terme de coques "liées" pour désigner les modes de liaison exclusifs des mers de l'Asie du Sud-Est, en partie observés aujourd'hui encore, mais dont les techniques plus anciennes -comme leur évolution progressive- ont pu être documentées sur les deux derniers millénaires, à partir de données textuelles et archéologiques: les ligatures qui y tiennent les bordés assemblés entre eux sont discontinues (en anglais, on parlera de stitches et de stitched-plank technique, par opposition à sewn-plank) ; les bordés ne sont pas perforés sur toute leur longueur, mais seulement par des paires d'orifices forés à intervalles réguliers; sauf rares exceptions, ces orifices traversent les bordés de leur face intérieure à leurs cans : les ligatures sont donc invisibles du dehors de la coque. En Asie du Sud-Est, les fibres les plus couramment utilisées de nos jours encore sont celles du palmier à sucre (Arenga pinnata, le ijok des Malais, le cabo negro des Tagalog); elles ont été retrouvées et identifiées par des botanistes à partir de cordes restées sur les épaves datant du début de notre ère.

Ces faits établissent d'emblée une première distinction entre les coques «cousues » de l'océan Indien et les coques «liées» des mers de l'Asie du Sud-Est. Toutefois, la description des techniques d'assemblage des divers éléments structurels des bateaux de l'Asie du Sud-Est doit être également accompagnée de données complémentaires, qui vont derechef les distinguer des techniques pratiquées communément dans l'océan Indien ${ }^{11}$. En effet, seuls les peuples de l'Asie du Sud-Est insulaire associent intimement la ligature des bordés entre eux à un ensemble technique plus complexe. Des blocs de bois rectangulaires sont réservés à intervalles réguliers sur la face interne des bordés lorsque ceux-ci sont formés à l'herminette -ils sont en général désignés par le terme austronésien tambuku, qui peut, entre autres, décrire un sorte de bouton servant à lier deux pièces de tissu ensemble-; ces tambuku sont perforés et permettent donc de lier le bordage, d'une part aux couples, et de l'autre à des rangées de baux, qui viennent toujours s'appuyer sur eux. Ces baux sont à leur tour liés entre eux et à la quille par des 
torsades verticales de cordages. La structure ainsi obtenue grâce à ces trois éléments constitutifs essentiels du bateau -coque, couples et baux- forme donc un tout cohérent, maintenu en compression par l'ensemble de ces ligatures ${ }^{12}$.

Ce mode d'assemblage par ligature fut d'abord identifié par les ethnographes en fonction des seuls liens qui, sur les tambuku (« lugs»), unissent les baux et les couples aux bordés ; la ligature des bordés entre eux, que l'on observera plus tard sur les seuls sites archéologiques, n'était pas encore prise en compte. Il est désigné en anglais par l'expression lashed-lug technique, que l'on pourrait rendre en français, de façon plus complète, par "technique des coques liées", qui englobe l'ensemble des ligatures: bordés entre eux et bordage aux couples et aux baux.

Des données textuelles - dont les plus anciennes remontent au IV ${ }^{e}$ siècle de notre èreet celles obtenues lors de la fouille contrôlée ou le sauvetage d'une douzaine d'épaves de coques liées datant des $\mathrm{III}^{\mathrm{e}}$ au XII ${ }^{\mathrm{e}}$ siècles de l'ère chrétienne ont permis, en les combinant aux observations ethnographiques, de comprendre l'évolution au long cours de cette tradition technique propre à l'Asie du Sud-Est. Si aucune des embarcations modernes observées par les voyageurs modernes et les ethnologues n'est de très grande taille (jamais plus d'une douzaine de mètres), il est bon de rappeler que les fouilles archéologiques ont livré à Sumatra des fragments de plusieurs navires de très fort tonnage, datés respectivement des $\mathrm{V}^{\mathrm{e}}-\mathrm{VII}{ }^{\mathrm{e}}$ siècles et du $\mathrm{XII}^{\mathrm{e}}$ siècle, qui pouvaient atteindre de 20 à $30 \mathrm{~m}$ de long; cela confirme des textes chinois du IVe et du VIII ${ }^{e}$ siècles qui parlent de coques « liées » de quelque $50 \mathrm{~m}$ de long et pouvant transporter jusqu'à 500 passagers ${ }^{13}$.

36 L'ensemble des données disponibles à ce jour sur les coques liées d'Asie du Sud-Est permet d'appréhender, déjà avec un bon degré de certitude, certaines évolutions de cette tradition. Toutes les coques les plus anciennes, qui datent du début du premier millénaire de notre ère, ont leurs bordages assemblés au moyen de rares chevilles et de ligatures nombreuses; les tambuku auxquels devaient être liés les couples et les baux sont toujours présents sur la face interne de leurs bordés comme de leur quille ${ }^{14}$. Sur certaines épaves plus récentes, les ligatures entre bordés se font moins nombreuses, alors que le nombre de chevilles augmente en proportion; on observe alors parfois des contre-chevilles, qui empêchent ainsi les bordés de s'écarter l'un de l'autre, et remplacent donc fonctionnellement les ligatures entre bordés, lesquelles tendent à disparaître (on a vu que ces contre-chevilles étaient utilisées aux Maldives). Ces ligatures sont absentes de certaines épaves plus tardives de Sumatra; elles ne sont pas mentionnées dans un texte du XVII ${ }^{\mathrm{e}}$ siècle relatant en grand détail la construction d'un baranghay philippin à coque liée, pas plus que dans les diverses descriptions ethnographiques des embarcations de l'Est indonésien ; les chevilles y sont en revanche très rapprochées. Les ligatures sont désormais limitées aux seules liaisons entre la coque d'une part et les couples et les baux d'autre part.

Cette évolution de la technique -avec abandon des ligatures entre bordés mais maintien des blocs liés aux couples et aux baux- est observée aujourd'hui sur les seules embarcations de l'Est indonésien. En revanche, sur les grands navires marchands affrétés par les royaumes des côtes occidentales de l'Asie du Sud-Est pendant la première moitié du deuxième millénaire de notre ère, il semble qu'une autre évolution ait pris place, qui n'est malheureusement pas encore attestée dans un contexte archéologique : les blocs (tambuku) y disparaissent totalement, avec les rangées de baux transversaux et l'ensemble des ligatures; les coques sont entièrement maintenues en 
place par des chevilles en bois, qui joignent les bordés entre eux et la coque aux couples. Dans les années soixante-dix, la plus grande partie de l'importante flotte marchande à voile de l'Indonésie était encore construire selon cette dernière technique ${ }^{15}$.

Il nous reste à dresser les parallèles entre les traditions techniques attestées en Asie du Sud-Est et aux Maldives. Quant aux éléments structurels, on retrouve d'abord sans aucun doute possible, des deux côtés, les blocs en bois réservés sur la face interne des bordés, sur lesquels viennent s'appuyer et auxquels sont liés les couples et les rangées de baux. Aux Maldives, les baux de chaque rangée ne sont plus aujourd'hui rendus solidaires par des torsades de cordage comme en Asie du Sud-Est, mais ce rôle fonctionnel y est encore clairement assumé par de petites pièces verticales en bois (les " crampons" haki). Or, l'usage conjoint des blocs en bois et des ligatures de fibres végétales ou de tout autre mode de liaison qui permette de rendre la coque solidaire des couples et des baux, et ces derniers entre eux, forme un tout fonctionnel dont on a vu qu'il était propre en Asie aux traditions techniques des seuls peuples de l'Asie du Sud-Est.

39 Sachant que, à un stade antérieur, les charpentiers de marine d'Asie du Sud-Est faisaient appel à la ligature mutuelle des bordés, on obtient confirmation des indications textuelles et linguistiques signalées plus haut, selon lesquelles les bateaux des Maldives auraient été, eux aussi, construits par le passé avec des bordés liés entre eux. L'ensemble du complexe technique donnant corps aux «coques liées » d'Asie du Sud-Est se trouve donc transposé aux Maldives, sans ambiguïté aucune me semble-t-il.

Le corpus de données disponibles n'ayant pas aux Maldives la même profondeur diachronique, il est impossible d'y suivre précisément l'évolution des mêmes paramètres techniques que ceux observés en Asie du Sud-Est. Ainsi, on a vu qu'en Asie du Sud-Est le renforcement du chevillage chasse progressivement les ligatures des bordés entre eux; ces données ne sont pas disponibles aux Maldives, où les seules informations sur la ligature des bordés entre eux proviennent de trois brèves mentions textuelles des $\mathrm{XV}^{\mathrm{e}}$ et $\mathrm{XVI}{ }^{\mathrm{e}}$ siècles. En revanche, j'ai pu constater aux Maldives, pratiquement de visu, la disparition progressive des blocs de bois, remplacés par des couples rivetés à la coque. Ce processus pourrait bien expliquer une évolution parallèle, mais encore mal documentée, notée plus haut, vers les coques exclusivement chevillées en bois des grands navires des royaumes marchands des mers de l'Asie du Sud-Est (navires qui pouvaient atteindre les 500 tonnes de port en brut). En Indonésie comme aux Maldives, dans cette phase de l'évolution technique, les bordés étaient joints aux couples par des chevilles tronconiques, bloquées en place par l'insertion depuis l'extérieur de la coque d'un petit coin en bois (et remplacées aujourd'hui aux Maldives par des rivets, et en Indonésie par des boulons et leurs écrous, mieux à même de résister aux vibrations des moteurs).

41 Pour mieux mettre en relief ces parallèles techniques, notons en outre que certains petits navires traditionnels à coque entièrement chevillée de Java-Est et de Madura (tel le golekkan), s'ils ne gardent aucune trace de tambuku sur la face interne de leurs bordés, n'en sont pas moins construits avec des rangées de baux qui sont chevillés aux couples et au bordage. Ils pourraient donc représenter un stade intermédiaire de l'évolution de cette technique vers des coques conçues sans rangées de baux, stade à tous égards comparable à celui observé sur les dhoni les plus récents des Maldives, où ces mêmes rangées de baux disparaissent, comme on l'a remarqué plus haut. 

l'assemblage des bordés successifs qui constituent la coque des dhoni. Le respect de la procédure, on l'a vu, permet d'assurer, sans calfatage ultérieur, l'étanchéité de la structure par son maintien permanent en compression, jusqu'à ce que l'ensemble achevé assure par lui-même les forces essentielles à son bon fonctionnement. Une séquence en tous points comparable a été décrite aux Philippines au XVII siècle et, plus récemment, aux Moluques, à propos de navires comportant encore des bordés avec des blocs en bois (tambuku) auxquels sont liées les rangées de baux et les couples. Les analogies sont frappantes, alors que le cocotier ne fournit aucun des matériaux de construction: mêmes essais successifs pour ajuster la forme des bordés débités à l'herminette, avec usage de chevilles provisoires, du trusquin et du noir de suie ; même insertion de fibres végétales entre les virures avant l'assemblage final, pour obtenir l'étanchéité de la coque sans calfatage ; mêmes cordages enserrant la coque en cours de construction, tendus par des tourniquets pour la maintenir en compression. Lors de recherches effectuées dans les années soixante-dix sur les chantiers navals de l'Indonésie occidentale, j'ai pu observer encore la même séquence technique, bien que les tambuku n'y fussent plus employés depuis longtemps ${ }^{16}$ (photo 11).

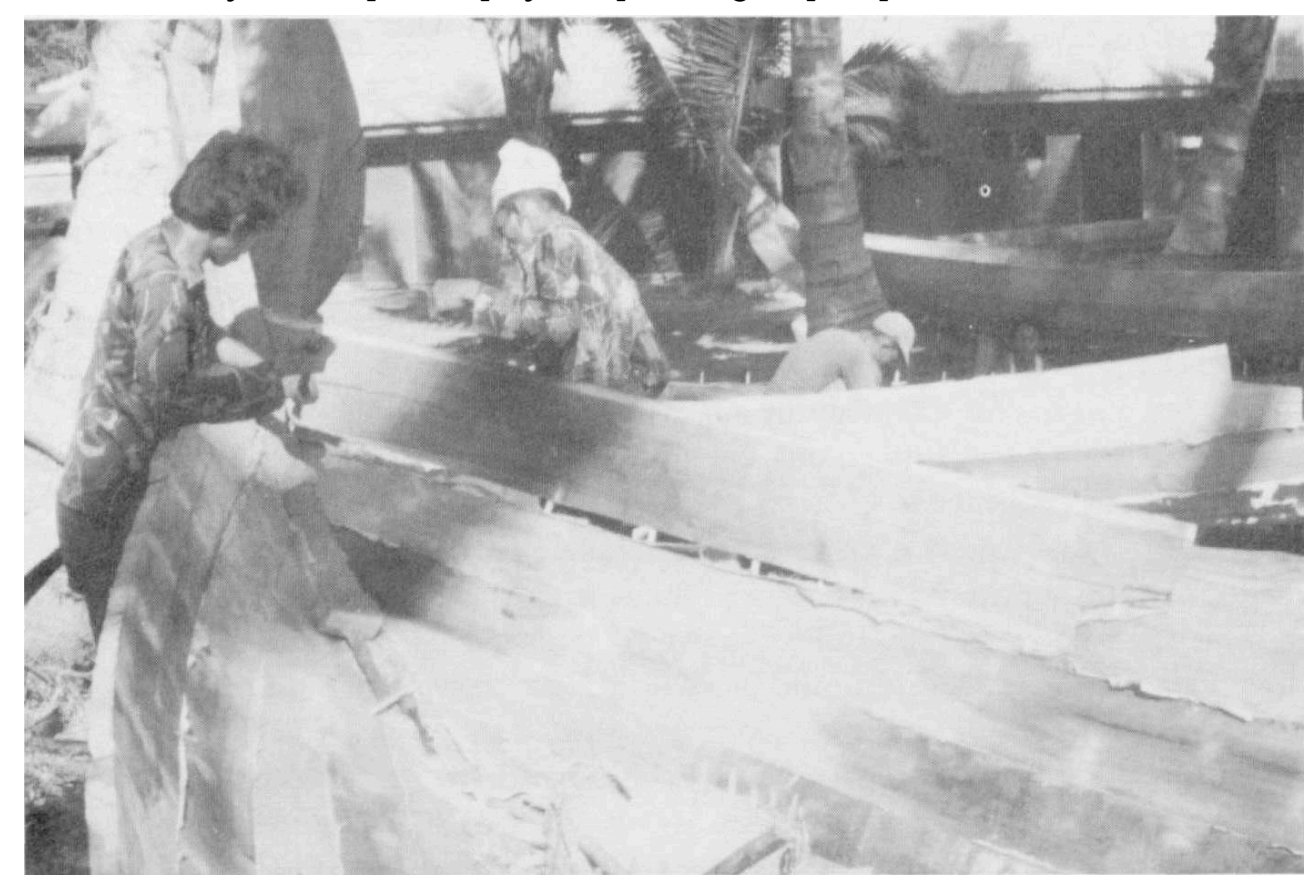

Photo 11. Processus d'assemblage de la coque d'un perahu pacaron à Pakisan (Java-Est) : le bordé est mis en place après plusieurs essais ; les chevilles sont définitives

\section{Conclusion}

Les analogies entre les deux traditions techniques me paraissant ainsi établies, il reste à tenter de les expliquer. On a bien constaté depuis longtemps que certaines des techniques de construction navale développées par les peuples austronésiens, comme les balanciers, ont essaimé au long des côtes de l'océan Indien jusqu'en Afrique de l'Est et à Madagascar. On a toujours invoqué pour l'expliquer des "migrations" austronésiennes qui auraient contribué au peuplement de Madagascar, à une époque 
"préhistorique » indéterminée ${ }^{17}$. Il s'agit en outre, dans tous les cas, de bateaux de taille et de complexité très réduites, pour l'essentiel de pirogues monoxyles.

Or, aux Maldives, la tradition technique apparaît autrement sophistiquée : il s'agit de coques entièrement construites avec des bordés, pouvant compter jusqu'à une vingtaine de virures, qui font intervenir des principes structurels complexes; et cette tradition technique n'a été repérée nulle part ailleurs dans la zone de diffusion du balancier austronésien sur l'océan Indien (en dehors bien sûr des mers d'Indonésie dont elle est originaire) ${ }^{18}$.

Des travaux linguistiques récents prouvent que les contacts entre l'Asie du Sud-Est insulaire et Madagascar se sont prolongés à l'époque historique, au moins jusqu'aux premiers siècles du deuxième millénaire de notre ère (Adelaar 1989, 1991, 1995). J'ai pour ma part tenté de démontrer, en me fondant sur les sources historiques disponibles, que non seulement la fréquentation des Maldives par les navires de JavaOuest était bien attestée au début du XVI ${ }^{e}$ siècle, mais que les navires marchands des Malais et des Javanais ont certainement continué à fréquenter l'ensemble de l'océan Indien, jusqu'à Madagascar, jusqu'aux $\mathrm{XIV}^{\mathrm{e}}$ et $\mathrm{XV}^{\mathrm{e}}$ siècles; et qu'ils l'ont très probablement fait par une voie méridionale, avec une étape aux Maldives (lorsqu'ils voyageaient vers le ponant, la route du retour devant obligatoirement passer par le nord de l'océan Indien) ${ }^{19}$.

Les navires des royaumes marchands des mers occidentales de l'Asie du Sud-Est, on l'a $\mathrm{vu}$, étaient à l'époque de tonnage considérable; leur construction et leur fonctionnement faisaient donc appel à des processus très complexes, bien plus sophistiqués que ceux impliqués par la construction de simples pirogues à balancier.

Si l'on en croit les seules sources historiques - puisque aucun de ces navires n'a encore été mis au jour dans un contexte archéologique-, il semble bien que ces navires destinés aux commerce hauturier aient déjà été, aux $\mathrm{XV}^{\mathrm{e}}$ et $\mathrm{XVI}^{\mathrm{e}}$, siècles entièrement chevillés, et par suite, que les liens en fibres végétales aient alors cessé d'être utilisés pour assembler les éléments structurels de leur coque. Mais on sait que des navires de fort tonnage faisant appel à la technique des «blocs liés » sont encore attestés sur le site marchand de Kota Cina, à Sumatra, vers le XII e siècle (Manguin 1980, 1984, 1989, 1993). Du moins a-t-on ainsi la preuve que les relations avec les royaumes marchands indonésiens étaient régulières pendant cette période de transition entre coques liées et coques chevillées. Rien ne permet encore, malheureusement, de discerner le début des contacts entre l'Asie du Sud-Est et les Maldives -pas plus d'ailleurs que les débuts des contacts des peuples de parler austronésien avec Madagascar. Le fait que les Maldives aient encore connu aux $\mathrm{XV}^{\mathrm{e}}$ et $\mathrm{XVI}^{\mathrm{e}}$ siècles la tradition des bordés liés entre eux, comme on l'a vu plus haut, laisse à penser que la transmission de cette technique a pris place à un moment où celle-ci était encore largement pratiquée sur les plus gros navires de l'Asie du Sud-Est, à savoir au cours du premier millénaire de notre ère.

Une deuxième hypothèse de travail permettrait aussi d'expliquer les analogies entre les deux traditions techniques. Cette explication, plus générale, n'est d'ailleurs pas exclusive de la première. Tant les traditions techniques des Maldives que celles de l'Asie du Sud-Est ont pu appartenir à un ensemble plus vaste, englobant la totalité de la partie orientale de l'océan Indien, avec peut-être la côte est de l'Inde. Je l'ai déjà proposée à d'autres occasions, en me fondant sur l'existence, dès le début du premier millénaire, d'un corpus partagé de vocabulaire nautique; mais la stagnation, depuis lors, des recherches sur les anciennes techniques nautiques indiennes et, en particulier, 
l'absence complète de données issues de l'archéologie nautique pour le golfe du Bengale (hors les navires européens), interdit pour l'instant de reconstituer les principes structurels qui régissent la construction des anciens navires indiens, et par là même de comprendre le pourquoi de l'usage de ces termes austronésiens dans un contexte indien (Manguin 1985a : 14-15, 1995 : 191-192). Or, on sait que le vocabulaire nautique voyage plus vite et plus loin, le long des réseaux marchands, que les techniques auxquelles il est associé sur son lieu d'origine. Contrairement à la première des hypothèses, qui est étayée par des observations techniques détaillées, celle-ci demeure très ténue.

Il me semblerait donc prématuré de vouloir présenter aujourd'hui une explication directe et précise des analogies notées dans cet article. Tout au plus doit-on les attribuer, en termes généraux, aux contacts historiques attestés des charpentiers de marine des Maldives avec les marins et les marchands de l'Asie du Sud-Est insulaire qui ont fréquenté très régulièrement l'ensemble de l'océan Indien pendant les deux premiers millénaires de notre ère, pour le moins. Ils naviguaient sur des navires de fort tonnage, construits selon des techniques qui leur étaient propres et dont on a retrouvé l'écho aux Maldives.

\section{BIBLIOGRAPHIE}

Adelaar, K.A., 1989, « Malay influences on Malagasy : linguistic and culture-historical inferences », Oceanic Linguistics 28 (1) : 1-46.

- 1991, « New ideas on the early history of Malagasy », pp. 1-22, in H. Steinhauer (ed.), Papers in Austronesian Linguistics. Canberra : Pacific Linguistics A-81.

- 1995, « Asian roots of Malagasy : A linguistic perspective », Bijdragen tot de Taal-, Land- en Volkenkunde van de Koninklijk Instituut 151 (3) : 325-356.

Barnes, R.H., 1985, « Whaling vessels of Indonesia », pp. 345-366, in S. McGrail and E. Kentley (eds.), Sewn Planked Boats. Archaeological and Ethnographic Papers Based on those Presented to a Conference at Greenwich in November, 1984. London : National Maritime Museum, Greenwich, Archaeological Series $n^{\circ} 10$ (BAR International Series $n^{\circ} 276$ ).

Bell, H.C.P., 1940, The Maldive Islands : Monograph on the History, Archaeology and Epigraphy. Colombo : Ceylon Government Press.

Block, G., 1992, « Island craft : gus block looks at traditional boatbuilding in the Maldives », Classic Boat, February : 22-26.

Clark, P. J. Green et T. Vosmer, 1993, « The Butuan Two boat known as a balangay in the National Museum, Manila, Philippines », International Journal of Nautical Archaeology 22 (2) : 143-159.

Correia, Gaspar, 1858-66, Lendas da India. Lisboa (8 vols).

Hornell, James, 1920, «The common origin of the outrigger canoes of Madagascar and East Africa », Man 20 (67) : 134-139.

- 1935, « Constructional parallels in Scandinavian and oceanic Boat Construction », Mariner's Mirror 21 : 411-427. 
- 1942, « Outrigger devices : distribution and Origins », Journal of the Polynesian Society 52 : 91-100. - 1946, Water Transport : Origins \& Early Evolution. London (réimpression, Londres 1970).

Horridge, G.A., 1978, The Design of Planked Boats of the Moluccas. London : National Maritime Museum (Maritime Monographs and Reports, $n^{\circ} 38$ ).

- 1982, The lashed-lug boat of the Eastern Archipelagoes, the Alcina MS and the Lomblen Whaling Boats. London : National Maritime Museum (Maritime Museum Monographs and Reports, $n^{\circ} 54$ ).

Koechlin, Bernard, 1979, « Notes sur l'histoire et le navire long-courrier, odi, aujourd'hui disparu, des Maldives », Archipel $18: 283-300$.

Maloney, C., 1980, People of the Maldive Islands. Madras : Orient Longmans.

Manguin, Pierre-Yves, 1980, " The Southeast Asian ship : an historical approach », Journal of Southeast Asian Studies $11: 253-269$.

- 1984, « Relationship and cross-Influence between South-East Asian and Chinese shipbuilding traditions ", pp. 197-212, in Final Report. SPAFA Workshop on Shipping and Trade Networks in SouthEast Asia, 1984. Bangkok : SPAFA.

- 1985, « Sewn-plank craft of South-East Asia. A preliminary survey », pp. 319-343, in S. McGrail and E. Kentley (eds.), Sewn planked boats. Archaeological and Ethnographic Papers Based on those Presented to a Conference at Greenwich in November, 1984. London : National Maritime Museum, Greenwich, Archaeological Series $n^{\circ} 10$ (BAR International Series ${ }^{\circ} 276$ ).

- 1985a, « Late medieval Asian shipbuilding in the Indian Ocean : A reappraisal », Moyen Orient \& Ocean Indien 2(2) : 1-30.

- 1989, « The trading ships of Insular Southeast Asia : new evidence from Indonesian archaeological sites ", pp. 200-220, in Proceedings, Pertemuan Ilmiah Arkeologi V, Yogyakarta 1989. Jakarta : Ikatan Ahli Arkeologi Indonesia, vol.1.

- 1993, «Trading ships of the South China Sea : Shipbuilding techniques and their role in the history of the development of Asian trade networks ", Journal of Economic and Social History of the Orient $36: 253-280$.

- 1993a, «Pre-modern Southeast Asian shipping in the Indian Ocean : the Maldives connection », communication non publiée présentée à la Conference de l'Association internationale d'Histoire maritime (Fremantle).

- 1995, « Southeast Asian shipping in the Indian Ocean during the 1st millennium AD », pp. 181-198, in H.P. Ray and J.-F. Salles (eds), Tradition and archaeology. Early Maritime Contacts in the Indian Ocean. Lyon/New Delhi : Manohar/Maison de l'Orient méditerranéen/ NISTADS.

Maniku, Hassan Ahmed, 1989, « Filaaveli », Vanavaru 4 : 1-51.

Millar, K., 1993, « Preliminary report on observations made into techniques and traditions of Maldivian shipbuilding », Bulletin of the Australian Institute of Maritime History 17(1) : 9-16.

Mills, J.V.G. (ed.), 1970, Ma Huan. Ying-Yai Sheng-Lan : "The Overall Survey of the Ocean's Shores » (1433). Cambridge : Hakluyt Society (extra series).

Palmer, C., 1986, Preliminary Investigation of the Potential for the Use of Coconut Timber for Artisanal Fishing Boat Building. London : Overseas Development Agency (ODA Research Scheme R4168).

Scott, W.H., 1982, « Boatbuilding and Seamanship in Classic Philippine Society », Philippine Studies $30: 335-376$.

Skjølsvold, A., 1991, Archaeological Test-excavations on the Maldive Islands. Oslo : The Kon-Tiki Museum (Occasional papers $\mathrm{n}^{\circ} 2$ ). 


\section{NOTES}

2. Cette mission a été effectuée, en compagnie du Dr. Lotika Varadarajan, dans le cadre du programme "Indigenous Traditions of Indian Navigation », sous l'égide du Heras Institute of Indian History and Culture de Bombay et du National Institute of Scientific, Technical and Development Studies (Centre for Scientific and Industrial Research), New Delhi ; l'École française d'Extrême-Orient a contribué à son financement ; le Gouvernement des Maldives a aimablement fourni à la mission un interprète et un dhoni motorisé pour la durée de la mission, lui accordant la permission de séjourner dans des atolls où les visiteurs étrangers ne sont normalement pas admis. Je voudrais exprimer ici ma gratitude à M. Hassan Ahmed Maniku, de Male, qui a généreusement partagé ses connaissances approfondies de la culture des Maldives et qui nous a mis en contact avec nombre de personnes au fait des questions de navigation traditionnelle. Un premier état des résultats de cette mission figure dans P.-Y. Manguin, Summary report on a survey of traditional shipyards in the Maldives, Paris/New Delhi : EFEO/NISTADS, 1988, non publié, et dans une communication restée elle aussi inédite, présentée sous le titre de «Pre-modern Southeast Asian shipping in the Indian Ocean : the Maldives connection ", à la Conférence de la Commission internationale d'Histoire maritime, tenue à Fremantle en 1993 ; c'est une partie de cette dernière communication qui est ici publiée, après mise à jour et révisions (Manguin 1993a).

3. On se reportera en particulier à la partie archéologique de l'ouvrage de H.C.P. Bell (1940); des sondages ont été menés en 1983 par un équipe Scandinave (Skjølsvold 1991).

4. Fort peu de travaux d'ensemble sérieux sont consacrés aux Maldives: pour une première introduction à cet archipel, on lira avec profit le grand classique de H.C.P. Bell (1940) et la monographie plus générale et plus récente de C. Maloney (1980).

5. À ma connaissance, seules deux études techniques sur les dhoni ont été à ce jour publiées : Karen Millar (1993) a donné un rapport préliminaire sur une mission effectuée pour le Western Australian Maritime Museum en 1992, qui arrive largement aux mêmes conclusions que moi sur les analogies sud-est asiatiques de la tradition des Maldives, et le bref travail de Gus Block (1992), qui était chargé à la fin des années quatre-vingt d'une mission d'expertise du BIT auprès du State Shipyard d'Alifushi. Les dhoni ont commencé à être motorisés dans les années soixante-dix. Le terme générique dhoni sera qualifié pour en définir le type, en fonction de l'usage qui en est fait (ainsi, les bateaux dont nous avons observé la construction étaient pour l'essentiel des maas dhoni. c'est-à-dire des "bateaux [pour la pêche au] poisson", qui peuvent atteindre une quinzaine de mètres de long). Ce sont les seuls navires traditionnels à être aujourd'hui construits aux Maldives. Bien d'autres types de navires à voile existaient encore au début des années cinquante : bangala, batil, nau, odi, etc. (voir à leur propos les études d'ensemble sur les Maldives citées supra, Koechlin 1979 ou Millar 1993).

6. Colin Palmer (1986) a étudié l'usage qui a été fait de cette monocotylédone et de son bois pour la construction navale.

7. Pour confirmer mes notes de terrain, j'ai utilisé un précieux petit lexique des noms de plantes en dhivehi compilé par Hassan Ahmed Maniku (1989).

8. La fabrication et la commercialisation du « coir » sont réservées aux femmes. Au XVIe siècle, lorsque les sources portugaises décrivent pour la première fois les Maldives, ce cairo (mot portugais dérivé d'une langue dravidienne, qui a donné l'anglais "coir ») est déjà l'une des principales exportations des Maldives (Manguin 1985a : 4).

9. J'emploie librement dans cet article le terme "bau " pour désigner les pièces de bois transversales qui sont une partie essentielle de la structure (ils sont désignées par thwart, plutôt que beam, en anglais; en construction navale française, les baux sont plus spécifiquement les pièces transversales sur lesquelles reposent les ponts, ce qui n'est évidemment pas le cas des navires non pontés d'Asie du Sud-Est ou des Maldives). 
10. Une traduction anglaise du texte de Ma Huan est donnée dans Mills (1970: 146-147) ; le texte portugais de G. Correia est disponible dans son édition de 1858-66, vol. Il : 131-132; le texte de D. Barbosa est traduit en anglais dans Dames (1918, vol. Il : 103-104). Ces témoignages sur les techniques de construction navale, comme ceux portant sur l'ensemble de l'océan Indien à la même époque, sont rassemblés dans Manguin (1985a).

11. La technique des blocs de bois réservés à l'intérieur des bordés pour y lier couples et baux est attestée dans une seule autre région du monde, la Scandinavie (voir Hornell 1935, pour une première comparaison, étude réimprimée dans son célèbre Water transport, 1946). Quoi qu'en aient dit certains diffusionnistes à la Heine-Geldern, il ne peut s'agir que de deux innovations indépendantes. La technique de l'Asie du Sud-Est est passée, avec des variantes, dans le Pacifique ; il faut donc vraisemblablement l'associer à la culture des peuples austronésiens.

12. On lira avec profit les études techniquement très complètes d'Adrian Horridge $(1978,1982)$, qui a bien mis en évidence la cohérence des forces qui maintiennent en compréhension la structure de ces embarcations. Les données ethnographiques sur les coques de l'Asie du Sud-Est ont été rassemblées dans Manguin 1985 (où j'utilise encore à tort le terme commun de «sewnplank ", bordés " cousus", pour désigner ce mode d'assemblage). R. Barnes (1985) donne une description très complète des seuls bateaux à coque liée encore couramment utilisés dans l'Est indonésien pour la chasse à la baleine, et de l'usage qui en est fait.

13. Des données textuelles et archéologiques sur les coques liées des Philippines au XVIIe siècle ont d'abord été exploitées par W.H. Scott (1982). Les données historiques, complétées des résultats des fouilles archéologiques menées en Indonésie et en Malaisie, sont étudiées par Manguin (1980, 1985a. 1989); l'ensemble des données disponibles sur les coques liées de l'ancienne Asie du Sud-Est, textes, sites archéologiques et datations, est rassemblé et analysé dans Manguin (1995), où l'on trouvera aussi toutes les références bibliographiques sur la question. L'étude technique la plus exhaustive d'une des épaves du XIIIe siècle fouillées à Butuan (Mindanao), la seule d'Asie du Sud-Est à être assez complète pour permettre cette approche, a été publiée par Clark, Green et Vosmer (1993).

14. Cette quille, sur l'ensemble des épaves observées, n'a pas conservé la forme d'un monoxyle. Cependant, les observations ethnographiques confirment toutes que ce type de construction a évolué à partir d'un monoxyle dont les flancs ont été progressivement rehaussés de bordages, jusqu'à ce que le tronc évidé finisse par se transformer en quille de structure plus complexe.

15. Cette tradition des coques chevillées d'Asie du Sud-Est a été étudiée dans Manguin (1980, 1984, 1993).

16. Voir Scott (1982) pour les Philippines, et Horridge $(1978,1982)$ pour les Moluques; mon propre travail ethnographique est resté inédit.

17. Voir en particulier les travaux de lames Hornell (1920.1942), dont certains ont été repris dans Hornell (1946).

18. Cela ne signifie d'ailleurs nullement que des navires à bordés plus anciens, dont on sait qu'ils ont existé à Madagascar, n'appartenaient pas à cette tradition ; mais personne n'a encore tenté de reconstituer leurs techniques de construction.

19. Cette argumentation a été avancée dans la deuxième partie de la communication présentée à une conférence internationale, déjà citée, (Manguin 1993a), et qui sera publiée sous peu. 


\section{RÉSUMÉS}

Les techniques traditionnelles de construction navale ont été observées aux Maldives à la fin des années quatre-vingt. Le processus complet utilisé pour la construction de la coque d'un dhoni est décrit en détail, et les principes structuraux présidant à l'assemblage de ses divers éléments sont ensuite analysés. Des sources historiques sont invoquées pour confirmer les observations modernes qui tendent à indiquer que les bordés étaient auparavant liés entre eux au moyen de fibres végétales. Des analogies frappantes entre cette tradition technique et celle observée en Asie du Sud-Est sont ensuite décrites, tant en ce qui concerne les modes d'assemblage que dans l'évolution diachronique de ces techniques. En conclusion, pour expliquer ces analogies, deux hypothèses complémentaires sont proposées : les Maldives constituent une étape naturelle sur la route qui mène les peuples austronésiens de leurs îles d'origine à Madagascar ; en se fondant sur l'existence d'un vocabulaire nautique partagé, on peut aussi envisager l'existence ancienne d'une tradition technique commune à la partie orientale de l'océan Indien.

Traditional boatbuilding was observed in various atolls of the Maldives in the late 1980s. The full process of building the hull of a dhoni is described in some detail, and the structural principles that lay behind the assembling of its elements are analysed. Historical sources are called upon to confirm modern observations that indicate former lashing of the planks together with vegetal fibre. Striking analogies are then observed between Maldivian and Southeast Asian boatbuilding traditions, both in the present day assembling process and in the diachronic evolution of the techniques. As a conclusion, two complementary hypothesis are offered to explain such close similarities (and common differences with other known Indian Ocean traditions): the Maldives are a natural way point on a route leading the Austronesian people from their Southeast Asian homeland to Madagascar; on the basis of shared nautical vocabulary, it also appears that the eastern parts of the Indian Ocean could have shared, in early historic times, a single boatbuilding tradition.

Las técnicas tradicionales de construcción naval se estudiaron en las Maldivas a final de los ochenta. El proceso completo de elaboración del casco de un dhoni está descrito detalladamente ; luego se analizan los principios estructurales presidiendo al asemblaje de sus diversos elementos. Con ayuda de fuentes históricas se llega a confirmar que los tablazones estaban anteriormente atados entre sí por medio de fibras vegetales. En seguida se describen unas analogías sorprendentes, tanto en lo que concierne los modos de asemblaje como en la evolución diacrónica de los mismos. En forma de conclusión, se ofrecen dos hipótesis complementarias : las Maldivas constituyen una etapa natural en el recorrido que lleva a los pueblos austronesianos desde sus islas de origen hasta Madagascar ; basándose en la existencia de un vocabulario náutico compartido, también se puede considerar la existencia antigua de una tradición técnica común a la parte oriental del océano índico.

\section{INDEX}

Mots-clés : Asie du Sud-Est, Austronésiens, construction navale, dhoni, évolution technique, Indonésie, Maldives 
AUTEUR

PIERRE-YVES MANGUIN

École Française d'Extrême-Orient, 22 avenue du Président Wilson, 75116 Paris 